\title{
Building the Spine: The Vertebrate Segmentation Clock
}

\author{
O. PourquiÉ \\ Howard Hughes Medical Institute, Stowers Institute for Medical Research, \\ Kansas City, Missouri 64110
}

\begin{abstract}
One of the most striking characteristics of many animal and plant species is their organization in a series of periodically repeated anatomical modules. In animals, this particular patterning strategy of the body axis is termed segmentation, and it is observed in both vertebrates and invertebrates. Vertebrate segmentation has been associated with a molecular oscillator-the segmentation clock - whose existence had been predicted on theoretical grounds in the clock and wave-front model. The segmentation clock is proposed to generate pulses of signaling used for the positioning of segmental boundaries. Whereas several models have proposed that simple negative autoregulatory circuits involving the transcription repressors of the hairy and enhancer of split family constitute the clock pacemaker, recent microarray studies in mouse have identified a large network of oscillating signaling genes belonging to the Notch, Wnt, and FGF (fibroblast growth factor) pathways. Thus, significant progress has been made, but the molecular nature of the clockwork underlying the oscillator remains poorly understood. Few examples of oscillators exist in developmental biology, and the segmentation clock provides a unique model of periodic regulation in patterning.
\end{abstract}

\section{INTRODUCTION}

Segmentation has been an intense subject of study since the 19th century when it was first taken by Geoffroy Saint Hilaire as an argument to support the concept of the unity of the body plan among animals. In vertebrates, this particular mode of organization is especially conspicuous at the level of the serial arrangement of vertebrae in the spine. This segmental or metameric pattern is established during embryogenesis through the somitogenesis process (Pourquié 2001). Somites are epithelial blocks of mesoderm, containing the precursors of the vertebrae and the skeletal muscles that are formed rhythmically from the anterior tip of the presomitic mesoderm (PSM) on both sides of the embryonic axis. The rhythm of somite production is characteristic of the species and ranges from 30 minutes in zebra fish embryos to several hours in mammals. Somites form in a sequential anterior-to-posterior progression until a defined number, also characteristic of the species, is reached. This number ranges between approximately 30 in some fish and up to several hundred in snakes (Richardson et al. 1998).

\section{THE CLOCK AND WAVE-FRONT MODEL}

The 1970s was a particularly fertile period for theoretical biology and witnessed the birth of important conceptual models in developmental biology. Among the important models postulated at the time was the "clock and wave-front" model proposed by Jonathan Cooke and Chris Zeeman (1976). This model was inspired by the mathematical theory of Catastrophes originally developed by Rene Thom. It proposed that PSM cells forming a somite underwent an abrupt change in cellular properties that could be formalized by a particular type of mathematical catastrophe. Such a catastrophe can be simply represented as a bistable transition between two steady states, allowing an abrupt switch from one particular state to another (Goldbeter et al. 2007). To make the catastrophe periodic, Cooke and Zeeman had to postulate an oscillator controlling the response of PSM cells to the mechanism triggering the catastrophe. Following this pioneering work, a number of subsequent models were proposed, all differing in their modalities but, for many, also relying upon the conversion of a temporal oscillation into a spatial periodic pattern (for review, see Dale and Pourquié 2000; Kulesa et al. 2007). Predicting the behavior of such oscillatory systems is often beyond intuition and thus requires mathematical modeling (Pourquié and Goldbeter 2003).

\section{THE SEGMENTATION CLOCK OSCILLATOR}

The first evidence for the existence of an oscillatory process coupled to somitogenesis was provided by the illustration of the rhythmic expression of the c-hairyl mRNA in the chick embryo PSM (Palmeirim et al. 1997). During the formation of each somite, the PSM is swiped by a dynamic wave of c-hairyl mRNA expression. This wave of transcription activation does not, however, require any long-range signaling among PSM cells and proceeds even if the tissue is physically interrupted, suggesting that it is largely cell-autonomous. These transcriptional oscillations of c-hairyl occurring with the same periodicity as the somitogenesis process were proposed to reflect the existence of a molecular oscillator (termed the segmentation clock) acting in PSM cells as originally proposed in the clock and wave-front model. Experimental demonstration of the existence of the oscillator provided a striking example of the predictive value of such theoretical models. Subsequently, several other genes exhibiting such a cyclic behavior were identified in fish, frog, and mouse, suggesting that the oscillator is conserved in vertebrates (Holley et al. 2000; Jiang et al. 2000; 
Jouve et al. 2000; Bessho et al. 2001b; Li et al. 2003; Dale et al. 2006). These genes are now referred to as cyclic genes, and the vast majority belong to the Notch, Wnt, and FGF signaling pathways.

\section{FISH OSCILLATOR, TIME DELAY, AND NEGATIVE FEEDBACKS}

I first discuss the fish oscillator and its modeling as it is believed to be simpler than the amniote oscillator. In fish, all of the cyclic genes identified thus far belong to the Notch pathway and comprise the Notch downstream targets Herl and Her7, which are homologous to the chick hairyl, as well as to the Notch ligand DeltaC (Holley et al. 2000; Jiang et al. 2000; Henry et al. 2002; Oates and Ho 2002). Large genetic screens carried out in zebra fish have identified a few mutants in which somitogenesis is disrupted (van Eeden et al. 1996). Most of these mutants show alterations in genes that belong to the Notch pathway (Rida et al. 2004; Holley 2007). These observations have led to the proposal of a central role for Notch signaling in the control of the oscillations. On the basis of the results of gain-of-function and loss-of-function experiments involving these Notch-related genes in the zebra fish embryo, Lewis (2003) proposed a simple oscillator model. This model is based on a central role of the transcriptional repressors Her1 and Her7, which can establish a simple negative feedback loop by negatively regulating their own promoter. By postulating the existence of a time delay between the production of the transcription factor and its binding on its own promoter, Lewis showed that oscillations exhibiting a period consistent with that of zebra fish somite formation could be obtained. Thus, in fish, the segmentation clock pacemaker is considered to essentially rely on this simple negative autoregulatory circuit (Lewis and Ozbudak 2007). So far in fish, only genes of the Notch pathway have been shown to oscillate, and the overlap with the mammalian cyclic genes is limited, suggesting that the mechanism underlying the fish oscillator could be quite different from the amniote oscillator discussed below.

\section{THE AMNIOTE OSCILLATOR: AN OSCILLATING SIGNALING NETWORK}

In amniotes such as chick or mouse, the situation appears to be somewhat different from that described in fish. Whereas a majority of the cyclic genes initially identified also belong to the Notch pathway (Palmeirim et al. 1997; Forsberg et al. 1998; McGrew et al. 1998; Aulehla and Johnson 1999; Jouve et al. 2000; Bessho et al. 2003), different but overlapping repertoires of genes appear to be used between fish and amniotes. For instance, no oscillations of the gene lunatic fringe are observed in zebra fish (Prince et al. 2001), whereas oscillations of the hairy homologs are detected in both species (Palmeirim et al. 1997; Holley et al. 2000; Jouve et al. 2000; Bessho et al. 2001a; Henry et al. 2002; Oates and Ho 2002). However, unlike that in fish, several other pathways appear to exhibit oscillations in the mouse PSM. For instance, Axin2, a key negative feedback inhibitor acting in the Wnt pathway, was shown to exhibit oscillations in the mouse PSM (Aulehla et al. 2003). More recently, a microarray screen carried out in mouse led to a more complex picture of the mouse oscillator (Dequeant et al. 2006). Analysis of a microarray time series encompassing one period of the segmentation clock using Fourier analysis led to the identification of a large group of periodic genes expressed in the mouse PSM (Dequeant et al. 2006). The first 40 cyclic genes identified by this strategy fall into two clusters oscillating in opposite phase. One cluster contains known genes of the Notch pathway, such as Hes1, but also genes of the Notch pathway that had not been previously associated with the oscillator, such as Nrarp. In addition, this cluster also contains known downstream targets of the FGF pathway, such as Sprouty2, suggesting that the FGF pathway oscillates in phase with Notch signaling in the posterior PSM. Axin2 was found to oscillate in the cluster in opposite phase to the Notch/FGF cluster. The Axin2 cluster contains several other genes of the Wnt pathway including the inhibitors Dkkl and Dactl and the Wnt targets $c-m y c, S P 5$, or Tnfrsf19 (Dequeant et al. 2006 and references therein). Remarkably, the majority of the cyclic genes of the Wnt and Notch/FGF clusters are feedback inhibitors or targets of these pathways (Dequeant et al. 2006 and references therein). Thus, this microarray analysis increased by an order of magnitude the number of cyclic genes identified in the mouse but did not really challenge the biological coherence of the system. However, it suggests that rather than a set of simple negative feedback loops acting as the pacemaker of the system, the amniote segmentation clock relies on a complex network of signaling feedback inhibitors. This analysis further suggests that the role of the segmentation clock is to deliver coordinated pulses of Notch, FGF, and Wnt signaling that are in turn used for the appropriate patterning of the segments. How this periodic signaling is translated into a coordinated striped gene activation that defines the segmental domain and its boundaries is now becoming better understood (Morimoto et al. 2005; Saga 2007).

\section{COUPLING THE OSCILLATORS: SYNCHRONIZATION OF THE OSCILLATIONS IN THE PSM}

A remarkable property of the segmentation clock is the synchrony with which the oscillations occur in nearby cells, resulting in the smooth dynamic pattern of the transcription wave observed in the PSM. Experimental evidence now supports a role for cell-cell communication (Horikawa et al. 2006) and more specifically for the Notch signaling pathway in this synchronization process as first postulated in zebra fish (Jiang et al. 2000). In fish, a handful of segmentation mutants have been identified in the large genetic screens carried out during the 1990s (van Eeden et al. 1996). These mutants, which are almost all in Notch pathway components, show remarkably similar phenotypes with a variable number of anterior somites that form, followed by a disruption of the segmentation process (Holley 2007). Additionally, expression of the cyclic genes Herl and DeltaC in these mutants shows a typical salt-and-pepper pattern instead of the dynamic 
profile seen in wild-type embryos (Jiang et al. 2000). This led to the proposal that the role of Notch in PSM cells is to synchronize the oscillations, which are initiated by a different mechanism (Jiang et al. 2000). Hence, in the embryos mutant for the Notch pathway, oscillations are set initially and the first somites segment normally. However, because of the lack of Notch-dependent coupling, oscillations progressively drift out of synchrony, resulting in the progressive failure of segmentation observed in the mutants. The salt-and-pepper expression of the cyclic genes would reflect uncoordinated oscillations in PSM cells. Although a definitive demonstration of this hypothesis remains to be provided, recent transplantation experiments in zebra fish demonstrate that implanting cells from an embryo injected with morpholino against the Herl and Her7 genes, which result in Notch ligand overepression, can desynchronize the waves of cyclic gene expression, resulting in the shifting of somitic boundaries on the injected side (Horikawa et al. 2006; Ishimatsu et al. 2007). These experiments strongly argue in favor of the role of Notch signaling in the synchronization of oscillations among nearby cells. Mathematical modeling of the Notch extracellular loop connected to the fish $\mathrm{Her} 1 / \mathrm{Her} 7$ oscillator further supports the proposed role of this pathway in coupling oscillations in nearby cells (Lewis 2003). Accordingly, dissociating the PSM cells-a procedure expected to impair Notch signaling, which requires cell-cell interactions-from chick embryos rapidly results in a loss of synchronized oscillations (Maroto et al. 2005). Similarly, dissociation of mouse PSM cells and analysis of their oscillations using a real-time luciferase-based reporter fused to the Hesl promoter show that dissociated cells exhibit very chaotic oscillatory patterns (Masamizu et al. 2006). Together, these data argue in favor of the role of the Notch pathway in synchronization of the oscillations among neighboring cells, thus resulting in the smooth oscillatory waves observed in the vertebrate PSM.

\section{THE WAVE FRONT: TRANSLATING THE CLOCK PULSE INTO A SPATIAL PERIODIC ARRAY OF SEGMENTS}

The wave front or determination front was proposed to be defined by a gradient of FGF and Wnt signaling that regresses in concert with axis elongation (Dubrulle et al. 2001; Sawada et al. 2001; Aulehla et al. 2003). At a particular threshold of FGF and Wnt signaling (the determination front), cells of the PSM become competent to respond to the segmentation clock (Dubrulle and Pourquié 2004; Delfini et al. 2005). Upon receiving the clock signal, competent PSM cells simultaneously activate expression of genes such as Mesp 2 in a striped domain, hence defining the future segment (Saga 2007). The role of genes of the Mesp2 transcription factor family, that respond to the periodic clock signal, has been shown to be critical for defining the future segment (Morimoto et al. 2005). Mesp2 controls a complex morphogenetic program resulting in boundary formation and specification of anterior and posterior somite compartments, ultimately leading to formation of the morpholog- ical segmental units (Saga and Takeda 2001; Saga 2007).

The process of segmentation in the embryo was shown to be tightly coordinated with that of axis elongation through the formation of the FGF signaling gradient in the posterior PSM (Dubrulle and Pourquié 2004). Transcription of the $f g f 8$ mRNA is restricted to the precursors of the PSM in the tail bud, and it ceases when their descendents enter the posterior PSM. Thus, as the axis elongates, cells become located progressively more anteriorly in the PSM, and their content in $f g f 8$ mRNA progressively decays. This results in the establishment of a dynamic gradient of $f g f 8$ mRNA which is converted into a graded ligand distribution and FGF activity (Sawada et al. 2001; Dubrulle and Pourquié 2004; Delfini et al. 2005). A similar mechanism is assumed to be responsible for establishing the Wnt gradient (Aulehla and Herrmann 2004). The determination front is constantly displaced posteriorly as a result of the progressive decay of the FGF/Wnt mRNA and proteins in PSM cells, and the speed of this displacement defines the speed of somitogenesis progression along the anteroposterior axis. Concomitantly, new cells produced by the tail bud and strongly expressing FGF and Wnt ligands are constantly added to the posterior PSM, accompanying the posterior elongation movement of the tail bud. The FGF and Wnt pathways that define gradients in the PSM also oscillate in this tissue (Aulehla et al. 2003; Dale et al. 2006; Dequeant et al. 2006), therefore arguing that the clock and the wave front are tightly coupled. The gradients also likely have an important role in directly controlling the timing of the arrest of the clock.

\section{WHEN THE OSCILLATOR FAILS: CONGENITAL SCOLIOSIS}

In humans, severe disruptions of the segmentation pattern of the vertebrae are generally called congenital scoliosis (Erol et al. 2004). Congenital scoliosis is a rare deformity of the spine, occurring in 1-2 per 10,000 births. It can negatively impact health, with progressive deformity resulting in cardiopulmonary compromise or neurological deficits. In addition, congenital scoliosis is often associated with anomalies of other organ systems, most commonly the renal, neural, and cardiac systems (Turnpenny et al. 2007). Clinical management of these patients is problematic primarily because it is difficult to predict the long-term behavior or associated anomalies of the other organ systems. Most forms of congenital scoliosis are thought to be sporadic in nature, but in fact, little information on familial incidence is available. Thus far, traditional linkage analysis in families with individuals affected by congenital scoliosis has led to the identification of three genes, all associated with the segmentation clock. Mutation of the genes Dll3, Mesp2, and lunatic fringe was shown to lead to familial forms of spondylocostal dysostosis - a particular form of congenital scoliosis (Bulman et al. 2000; Whittock et al. 2004; Sparrow et al. 2006). The fact that all the genes associated with familial congenital scoliosis thus far are linked to the segmentation clock suggests that these anomalies result from defects in the somitogenesis process (Turnpenny et al. 
2007). This suggests that the segmentation clock oscillator also acts in human embryos to control segmentation. Studies of the somitogenesis process in mouse embryos point to a number of genes whose mutation results in phenotypes resembling human congenital scoliosis, thus providing interesting candidate genes that could carry mutations in the patients. Therefore, deciphering the segmentation clock mechanism in model organisms will certainly help improve our knowledge of these diseases, which in turn could lead to a better clinical management of these patients.

\section{CONCLUSIONS}

Studies of the segmentation clock oscillator in vertebrates have begun to shed light on the complex mechanism involved in generating the characteristic periodic pattern of the vertebrate body axis. Different molecular circuitries appear to have been established by amniotes and lower vertebrates. It remains possible that the oscillatory network identified thus far, which involves interlocked inhibitory feedback loops of the Notch, FGF, and Wnt pathways, merely reflects an output of a pacemaker that would be conserved in these different species. How general is the role of oscillators in generating periodic structures in metazoans remains to be explored. Other examples of spatial periodic structures generated by an oscillator include the well-characterized circadian pattern of sporulation in Neurospora crassa, which results in stripe formation (Dunlap and Loros 2004). The role of such oscillators in generating spatial periodicity in animal and vegetal species appears thus far to be limited to systems with polarized growth. No such clock has been identified in the fly embryo in which axial growth occurs after segmentation (Davis and Patel 1999). In contrast, other arthropods that exhibit a progressive mode of axis extension show a segmentation mode that appears to be related to that of vertebrate and hence, could involve a molecular oscillator. Therefore, the use of a traveling oscillator might reflect a widely used strategy of metazoans for generating periodic patterns.

\section{ACKNOWLEDGMENTS}

Work in the author's laboratory is supported by a National Institutes of Health grant (R02HD043158) and by Stowers Institute for Medical Research. O.P. is a Howard Hughes Medical Institute Investigator.

\section{REFERENCES}

Aulehla, A. and Herrmann B.G. 2004. Segmentation in vertebrates: Clock and gradient finally joined. Genes Dev. 18: 2060.

Aulehla A. and Johnson R.L. 1999. Dynamic expression of lunatic fringe suggests a link between notch signaling and an autonomous cellular oscillator driving somite segmentation. Dev. Biol. 207: 49.

Aulehla A., Wehrle C., Brand-Saberi B., Kemler R., Gossler A., Kanzler B., and Herrmann B.G. 2003. Wnt3a plays a major role in the segmentation clock controlling somitogenesis. Dev. Cell 4: 395 .

Bessho Y., Hirata H., Masamizu Y., and Kageyama R. 2003. Periodic repression by the bHLH factor Hes7 is an essential mechanism for the somite segmentation clock. Genes Dev. 17: 1451.

Bessho Y., Miyoshi G., Sakata R., and Kageyama R. 2001a. Hes 7: A bHLH-type repressor gene regulated by Notch and expressed in the presomitic mesoderm. Genes Cells 6: 175.

Bessho Y., Sakata R., Komatsu S., Shiota K., Yamada S., and Kageyama R. 2001b. Dynamic expression and essential functions of Hes7 in somite segmentation. Genes Dev. 15: 2642.

Bulman M.P., Kusumi K., Frayling T.M., McKeown C., Garrett C., Lander E.S., Krumlauf R., Hattersley A.T., Ellard S., and Turnpenny P.D. 2000. Mutations in the human delta homologue, DLL3, cause axial skeletal defects in spondylocostal dysostosis. Nat. Genet. 24: 438.

Cooke J. and Zeeman E.C. 1976. A clock and wavefront model for control of the number of repeated structures during animal morphogenesis. J. Theor. Biol. 58: 455.

Dale J.K. and Pourquié O. 2000. A clock-work somite. Bioessays 22: 72.

Dale J.K., Malapert P., Chal J., Vilhais-Neto G., Maroto M., Johnson T., Jayasinghe S., Trainor P., Herrmann B., and Pourquié O. 2006. Oscillations of the snail genes in the presomitic mesoderm coordinate segmental patterning and morphogenesis in vertebrate somitogenesis. Dev. Cell 10: 355.

Davis G.K. and Patel N.H. 1999. The origin and evolution of segmentation. Trends Cell Biol. 9: M68.

Delfini M.C., Dubrulle J., Malapert P., Chal J., and Pourquié O. 2005. Control of the segmentation process by graded MAPK/ERK activation in the chick embryo. Proc. Natl. Acad. Sci. 102: 11343

Dequeant M.L., Glynn E., Gaudenz K., Wahl M., Chen J., Mushegian A., and Pourquié O. 2006. A complex oscillating network of signaling genes underlies the mouse segmentation clock. Science 314: 1595.

Dubrulle J. and Pourquié O. 2004. Coupling segmentation to axis formation. Development 131: 5783

Dubrulle J., McGrew M.J., and Pourquié O. 2001. FGF signaling controls somite boundary position and regulates segmentation clock control of spatiotemporal Hox gene activation. Cell 106: 219.

Dunlap J.C. and Loros J.J. 2004. The Neurospora circadian system. J. Biol. Rhythms 19: 414.

Erol B., Tracy M.R., Dormans J.P., Zackai E.H., Maisenbacher M.K., O’Brien M.L., Turnpenny P.D., and Kusumi K. 2004. Congenital scoliosis and vertebral malformations: Characterization of segmental defects for genetic analysis. $J$. Pediatr. Orthop. 24: 674.

Forsberg H., Crozet F., and Brown N.A. 1998. Waves of mouse Lunatic fringe expression, in four-hour cycles at two-hour intervals, precede somite boundary formation. Curr. Biol. 8: 1027.

Goldbeter A., Gonze D., and Pourquié O. 2007. Sharp developmental thresholds defined through bistability by antagonistic gradients of retinoic acid and FGF signaling. Dev. Dyn. 236: 1495.

Henry C.A., Urban M.K., Dill K.K., Merlie J.P., Page M.F., Kimmel C.B., and Amacher S.L. 2002. Two linked hairy/Enhancer of split-related zebrafish genes, her1 and her7, function together to refine alternating somite boundaries. Development 129: 3693.

Holley S.A. 2007. The genetics and embryology of zebrafish metamerism. Dev. Dyn. 236: 1422.

Holley S.A., Geisler R., and Nüsslein-Volhard C. 2000. Control of her1 expression during zebrafish somitogenesis by a deltadependent oscillator and an independent wave-front activity. Genes Dev. 14: 1678.

Horikawa K., Ishimatsu K., Yoshimoto E., Kondo S., and Takeda H. 2006. Noise-resistant and synchronized oscillation of the segmentation clock. Nature 441: 719.

Ishimatsu K., Horikawa K., and Takeda H. 2007. Coupling cellular oscillators: A mechanism that maintains synchrony against developmental noise in the segmentation clock. Dev. Dyn. 236: 1416.

Jiang Y.J., Aerne B.L., Smithers L., Haddon C., Ish-Horowicz D., and Lewis J. 2000. Notch signalling and the synchronization of the somite segmentation clock. Nature 408: 475. 
Jouve C., Palmeirim I., Henrique D., Beckers J., Gossler A., IshHorowicz D., and Pourquié O. 2000. Notch signalling is required for cyclic expression of the hairy-like gene HES1 in the presomitic mesoderm. Development 127: 1421.

Kulesa P.M., Schnell S., Rudloff S., Baker R.E., and Maini P.K. 2007. From segment to somite: Segmentation to epithelialization analyzed within quantitative frameworks. Dev. Dyn. 236: 1392.

Lewis J. 2003. Autoinhibition with transcriptional delay: A simple mechanism for the zebrafish somitogenesis oscillator. Curr. Biol. 13: 1398

Lewis J. and Ozbudak E.M. 2007. Deciphering the somite segmentation clock: Beyond mutants and morphants. Dev. Dyn. 236: 1410 .

Li Y., Fenger U., Niehrs C., and Pollet N. 2003. Cyclic expression of esr9 gene in Xenopus presomitic mesoderm. Differentiation 71: 83 .

Maroto M., Dale J.K., Dequeant M.L., Petit A.C., and Pourquié O. 2005. Synchronised cycling gene oscillations in presomitic mesoderm cells require cell-cell contact. Int. J. Dev. Biol. 49: 309.

Masamizu Y., Ohtsuka T., Takashima Y., Nagahara H., Takenaka Y., Yoshikawa K., Okamura H., and Kageyama R. 2006. Real-time imaging of the somite segmentation clock: Revelation of unstable oscillators in the individual presomitic mesoderm cells. Proc. Natl. Acad. Sci. 103: 1313.

McGrew M.J., Dale J.K., Fraboulet S., and Pourquié O. 1998. The lunatic fringe gene is a target of the molecular clock linked to somite segmentation in avian embryos. Curr. Biol. 8: 979 .

Morimoto M., Takahashi Y., Endo M., and Saga Y. 2005. The Mesp2 transcription factor establishes segmental borders by suppressing Notch activity. Nature 435: 354.

Oates A.C. and Ho R.K. 2002. Hairy/E(spl)-related (Her) genes are central components of the segmentation oscillator and display redundancy with the Delta/Notch signaling pathway in the formation of anterior segmental boundaries in the zebrafish. Development 129: 2929.

Palmeirim I., Henrique D., Ish-Horowicz D., and Pourquié O. 1997. Avian hairy gene expression identifies a molecular clock linked to vertebrate segmentation and somitogenesiss. Cell 91: 639.
Pourquié O. 2001. Vertebrate somitogenesis. Annu. Rev. Cell Dev. Biol. 17: 311.

Pourquié O. and Goldbeter A. 2003. Segmentation clock: Insights from computational models. Curr. Biol. 13: R632.

Prince V.E., Holley S.A., Bally-Cuif L., Prabhakaran B., Oates A.C., Ho R.K., and Vogt T.F. 2001. Zebrafish lunatic fringe demarcates segmental boundaries. Mech. Dev. 105: 175.

Richardson M.K., Allen S.P., Wright G.M., Raynaud A., and Hanken J. 1998. Somite number and vertebrate evolution. Development 125: 151.

Rida P.C., Le Minh N., and Jiang Y.J. 2004. A Notch feeling of somite segmentation and beyond. Dev. Biol. 265: 2 .

Saga Y. 2007. Segmental border is defined by the key transcription factor Mesp2, by means of the suppression of notch activity. Dev. Dyn. 236: 1450.

Saga Y. and Takeda H. 2001. The making of the somite: Molecular events in vertebrate segmentation. Nat. Rev. Genet. 2: 835 .

Sawada A., Shinya M., Jiang Y.J., Kawakami A., Kuroiwa A., and Takeda H. 2001. Fgf/MAPK signalling is a crucial positional cue in somite boundary formation. Development 128: 4873.

Sparrow D.B., Chapman G., Wouters M.A., Whittock N.V., Ellard S., Fatkin D., Turnpenny P.D., Kusumi K., Sillence D., and Dunwoodie S.L. 2006. Mutation of the LUNATIC FRINGE gene in humans causes spondylocostal dysostosis with a severe vertebral phenotype. Am. J. Hum. Genet. 78: 28.

Turnpenny P.D., Alman B., Cornier A.S., Giampietro P.F., Offiah A., Tassy O., Pourquié O., Kusumi K., and Dunwoodie S. 2007. Abnormal vertebral segmentation and the notch signaling pathway in man. Dev. Dyn. 236: 1456

van Eeden F.J., Granato M., Schach U., Brand M., Furutani-Seiki M., Haffter P., Hammerschmidt M., Heisenberg C.P., Jiang Y.J., Kane D.A., Kelsh R.N., Mullins M.C., Odenthal J., Warga R.M., Allende M.L., Weinberg E.S., and NüssleinVolhard C. 1996. Mutations affecting somite formation and patterning in the zebrafish, Danio rerio. Development 123: 153.

Whittock N.V., Sparrow D.B., Wouters M.A., Sillence D., Ellard S., Dunwoodie S.L., and Turnpenny P.D. 2004. Mutated MESP2 causes spondylocostal dysostosis in humans. Am. J. Hum. Genet. 74: 1249. 


\section{$8_{\mathrm{CSH}}^{\infty} \mathrm{C}$ Cold Spring Harbor Symposia SYMPOSIA on Quantitative Biology}

\section{Building the Spine: The Vertebrate Segmentation Clock}

O. Pourquié

Cold Spring Harb Symp Quant Biol 2007 72: 445-449

Access the most recent version at doi:10.1101/sqb.2007.72.016

References This article cites 48 articles, 13 of which can be accessed free at: http://symposium.cshlp.org/content/72/445.full.html\#ref-list-1

\section{License}

Email Alerting

Receive free email alerts when new articles cite this article - sign up in the box at the Service top right corner of the article or click here.

To subscribe to Cold Spring Harbor Symposia on Quantitative Biology go to: http://symposium.cshlp.org/subscriptions 